\title{
Role of MRI in Differentiating Benign from Malignant Breast Lesions Using Dynamic Contrast Enhanced MRI and Diffusion Weighted MRI
}

Kunal Singh Ahluwalia ${ }^{1}$, Harneet Narula ${ }^{2}$, Amit Jain ${ }^{3}$, Anshul Arora ${ }^{4}$, Aditi Vohra ${ }^{5}$, Tanu Bansal ${ }^{6}$, Akshit Gakhar ${ }^{7}$

1, 2, 3, 4, 5, 6,7 Department of Radiodiagnosis, MM Institute of

Medical Sciences and Research, Mullana, Ambala, Haryana, India.

\section{ABSTRACT}

\section{BACKGROUND}

Breast cancer is the second most common cancer in Indian women. Dynamic contrast enhanced MRI (DCE-MRI) has improved specificity in characterising breast lesions. Diffusion weighted imaging can improve the sensitivity and specificity of MRI in the evaluation of breast lesions thus differentiating between benign and malignant breast lesions. The purpose of the study was to evaluate the role of diffusion weighted MRI and dynamic contrast enhanced MRI in differentiating benign from malignant breast lesions and to compare its findings with histopathological or fine needle aspiration cytology (FNAC) findings.

\section{METHODS}

A descriptive diagnostic study enrolled 30 female patients of palpable breast lumps with positive findings either on mammography or ultrasound. Ultrasonography was done on HD 15 (Philips Medical Systems, USA). This was followed by MRI which was done on MULTIVA 1.5 T using a dedicated breast array coil.

\section{RESULTS}

Fibroadenoma accounted for majority of benign lesions (4 / 10) while invasive ductal carcinoma (IDC) accounted for majority of malignant lesions (15 / 20). 7 / 10 benign lesions showed type I curve, while majority (15/20) of the malignant lesions showed type III curve. 8 / 10 benign breast lesions did not show restricted diffusion on diffusion weighted imaging (DWI) while all malignant lesions showed restricted diffusion on DWI. In our study, the mean apparent diffusion coefficient (ADC) value for benign and malignant lesions was $1.59 \times 10^{-3} \mathrm{~mm}^{2} / \mathrm{s}$ and $0.88 \times 10^{-}$ ${ }^{3} \mathrm{~mm}^{2} / \mathrm{s}$ respectively.

\section{CONCLUSIONS}

MR morphology, DCE-MRI and DWI are useful to characterise various breast lesions. MRI features of signal intensity of hypointensity on T2WI with other associated features of irregular shape, spiculate margins, heterogeneous enhancement on DCEMRI, type III dynamic curve and reduced ADC value are strong predictors of malignancy.

\section{KEY WORDS}

Breast MRI, DCE-MRI, DWI, Breast Carcinoma

\author{
Corresponding Author: \\ Anshul Arora, \\ Department of Radiodiagnosis, \\ MM Institute of Medical \\ Sciences and Research, \\ Mullana, Ambala, \\ Haryana, India. \\ E-mail: arora.ashu123@gmail.com
}

DOI: $10.14260 /$ jemds/2021/300

How to Cite This Article:

Ahluwalia KS, Narula H, Jain A, et al. Role of MRI in differentiating benign from malignant breast lesions using dynamic contrast enhanced MRI and diffusion weighted MRI. J Evolution Med Dent Sci 2021;10(19):1422-1428, DOI: $10.14260 /$ jemds/2021/300

Submission 07-01-2021,

Peer Review 26-02-2021,

Acceptance 02-03-2021,

Published 10-05-2021.

Copyright (C) 2021 Kunal Singh Ahluwalia et al. This is an open access article distributed under Creative Commons Attribution License [Attribution 4.0 International (CC BY 4.0)] 


\section{BACKGROUND}

Breast cancer is the second most common cancer in Indian women. According to the National Cancer Registry project report about 52,000 women develop breast cancer in India per year. It is a significant cause of worldwide morbidity and mortality.

Mammography is the most commonly used method and is the only currently known means of proven effectiveness especially in patients with non-palpable carcinoma.

Conventional mammography and ultrasound are known to have high false positive rates in the detection of breast malignancy ( $60-80 \%)$, resulting in unnecessary biopsies being performed. So, MR techniques have shown strong potential to improve the sensitivity and specificity in the diagnosis of breast cancer. ${ }^{1}$

Breast magnetic resonance imaging (MRI) is recommended by the American Cancer Society as an adjunct to mammography for screening women who are at high risk of developing breast cancer. ${ }^{2}$ MRI seems to be ideally useful for breast imaging due to its ability to depict excellent soft tissue contrast. On the contrary, contrast enhanced MRI and dynamic MRI have been found to be more accurate in the detection of malignancy within dense breast tissue, differentiation of malignancy versus scarring and also in detection of implants. In addition, MRI can also be used to assess axillary lymph node metastasis.

DCE-MRI of breast is a very sensitive method for detecting even small lesions which are not visualised by other methods.

Breast malignancies have variable vascularization patterns. These patterns are classified due to the internal enhancement pattern, distribution of the enhancement, and kinetic studies on DCE-MRI. According to BIRADS lexicon, kinetic curves are classified as exhibiting a "washout," "plateau," or "persistent" shape. Type 1, a persistent enhancing curve, which shows a persistent increase in signal intensity, is associated with benign lesions. Type 2, a plateau curve, which demonstrates a slow or rapid increase in the beginning and then exhibits a plateau, which can be indicative of malignant pathology. Type 3 is a washout curve, which demonstrates an initial increase followed by subsequent decrease in signal intensity approx. 2 minutes after injection, thus this curve is highly suggestive for malignancy. ${ }^{3}$

DWI has shown promise for the detection and characterization of breast cancer. Apparent diffusion coefficient (ADC) values allow quantification of diffusion signal and can facilitate in differentiating benign and malignant breast tumours as well as identifying early response in tumours undergoing preoperative treatment. ${ }^{4}$

Usually, DWI is performed using at least two b values. Theoretically, the error in ADC calculation can be reduced by using more $b$ values. However, the more $b$ values used, the longer the DWI sequence will be. Thus, there is no consensus as to how many and which $b$ values to be used in differentiating benign and malignant breast lesions using DWI. ${ }^{5}$

The purpose of the study was to evaluate the role of diffusion weighted MRI and dynamic contrast enhanced MRI in differentiating benign from malignant breast lesions and to compare the findings of diffusion weighted MRI and dynamic enhanced MRI with histopathological or FNAC findings.
METHODS

A descriptive diagnostic study was carried out in the Department of Radiodiagnosis between June 2018 and September 2020, M.M. Institute of Medical Sciences and Research, Mullana, Ambala. A total of 30 patients with palpable breast lump referred from various wards and outpatient departments of MMIMSR, Mullana were included in the study.

\section{Inclusion Criteria}

Patients with palpable breast lump with either positive findings on mammography or ultrasound.

\section{Exclusion Criteria}

1. Patients who had received treatment (post chemotherapy, post radiotherapy, post-surgical).

2. Patients with impaired renal function.

3. Patients with allergy to contrast medium.

4. Patients with cardiac pacemaker or another contraindication to MRI.

A complete history was taken at the time of presentation. A thorough clinical examination was carried out. Relevant laboratory investigations were noted. All patients were to undergo a film screen mammography.

\section{Equipment}

1. Ultrasonography (USG): HD 15 (Philips Medical Systems, USA) with convex and linear probes.

2. Magnetic resonance imaging (MRI) - Multiva 1.5 T MRI

Patients underwent MRI on a Multiva $1.5 \mathrm{~T}$ using a dedicated breast array coil. T1w, T2w, SPAIR / fat saturated T2 weighted images in appropriate imaging planes were acquired. Diffusion weighted images were obtained using $b$ values of 0 and 1000 and ADC value was calculated. Dynamic contrast enhanced MR was performed using fat suppressed 3D T1 weighted images after intravenous injection of gadolinium. Single pre contrast scan was followed by 4 post contrast scans which were obtained for a duration of $4 \mathrm{~min}$ 24 seconds. The conventional MR images and DW images were evaluated for the presence of a breast mass / lesion, its signal characteristics and diffusion restriction.

Time intensity curves (TIC) were generated from dynamic contrast enhanced images. Findings of MRI scan were recorded in the Performa attached and diagnosis was made. Findings of the MRI (diffusion weighted and dynamic contrast enhancement) was analysed and correlated with histopathological and FNAC findings to evaluate their use as a diagnostic modality.

\section{Statistical Analysis}

Data was described in terms of range; mean \pm standard deviation $( \pm \mathrm{SD})$, frequencies (number of cases) and relative frequencies (percentages) as appropriate. Comparison of quantitative variables between the study groups was done 
using Student t-test and ANOVA. For comparing categorical data, chi-square $(\chi 2)$ test was performed, and exact test was used when the expected frequency was less than 5 . Sensitivity, specificity, accuracy, positive predictive value and negative predictive value were also calculated. A probability value (P- value) less than 0.05 was considered statistically significant. All statistical calculations were done using (Statistical Package for the Social Science) SPSS 21 version (SPSS Inc., Chicago, IL, USA) statistical program for Microsoft Windows.

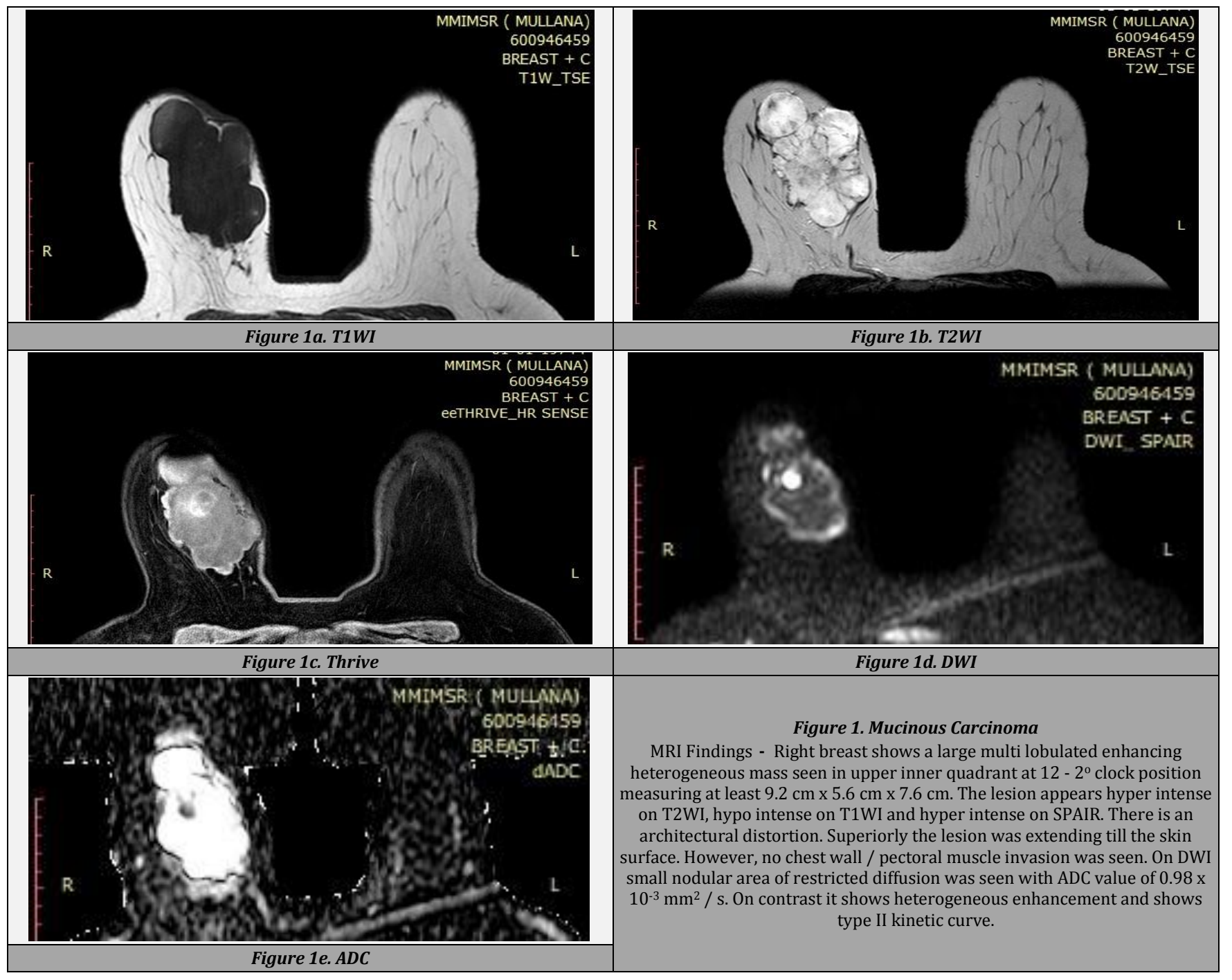

\section{RESULTS}

Out of the total 30 lesions, FNAC / biopsy analysis revealed 10 benign lesions (33.3\%) and 20 malignant lesions (66.7 $\%)$. Among the benign lesions, fibroadenoma was the most common pathology seen in $4 / 10$ cases (40\%), while intraductal carcinoma accounted for most of the malignant lesions seen in 15 / 20 cases (75\%). The study consisted of 30 females with youngest patient being 20 years old and oldest being 75 years old. All the patients with benign lesions were lesser than 40 years of age. Majority of the benign lesions in this study were either of type II or type III breast composition. Majority of the malignant breast lesions in this study were either of type I or type II breast composition.
Mammographic Features of Benign and Malignant Lesions According to Associated Features

- Architectural distortion was seen in only 2 out of 10 patients $(20 \%)$ with benign lesions on mammography as compared to 13 / 20 patients with malignant lesions (65\%) on mammography.

- Calcification was observed in 4 out of 10 cases with benign lesions ( $40 \%$ ) as compared to 19 out of 20 malignant lesions (95\%) seen on mammography.

- Skin thickening was seen in only 1 out of 10 benign lesions which was seen in a case of granulomatous mastitis while it was present in 9 out of 20 malignant breast lesions (45\%).

- Axillary lymphadenopathy was present in only 1 out of 10 benign lesions (10\%) as compared to 16 out of 20 malignant lesions (80\%)

- $\quad$ No benign lesions showed nipple retraction while it was present in 8 out of 20 malignant lesions. 


\begin{tabular}{|c|c|c|c|c|c|c|c|c|}
\hline & & & $\begin{array}{l}\text { nign } \\
=10 \text { ) }\end{array}$ & $\begin{array}{r}\text { Mal } \\
(\mathrm{N}\end{array}$ & $\begin{array}{l}\text { ignant } \\
=20 \text { ) }\end{array}$ & Total & $\begin{array}{c}\text { Chi- } \\
\text { Square } \\
\text { Value }\end{array}$ & P-Value \\
\hline \multirow{3}{*}{ Shape } & Irregular & 4 & $40.0 \%$ & 18 & $90.0 \%$ & 22 & \multirow{3}{*}{9.273} & \multirow{3}{*}{0.010} \\
\hline & Oval & 2 & $20.0 \%$ & 0 & $0.0 \%$ & 2 & & \\
\hline & Round & 4 & $40.0 \%$ & 2 & $10.0 \%$ & 6 & & \\
\hline \multirow{5}{*}{ Margin } & Circumscribed & 5 & $50.0 \%$ & 0 & $0.0 \%$ & 5 & \multirow{5}{*}{23.000} & \multirow{5}{*}{0.001} \\
\hline & Indistinct & 2 & $20.0 \%$ & 7 & $35.0 \%$ & 9 & & \\
\hline & Multilobulated & 0 & $0.0 \%$ & 2 & $10.0 \%$ & 2 & & \\
\hline & Obscured & 3 & $30 \%$ & 0 & $0.0 \%$ & 2 & & \\
\hline & Spiculated & 0 & $0.0 \%$ & 11 & $55.0 \%$ & 11 & & \\
\hline \multirow[b]{2}{*}{ Density } & High & 2 & $\begin{array}{c}20.0 \\
\%\end{array}$ & 18 & $\begin{array}{c}90.0 \\
\%\end{array}$ & 20 & \multirow[b]{2}{*}{17.400} & \multirow[b]{2}{*}{0.0001} \\
\hline & Iso & 2 & $\begin{array}{c}\% \\
20.0 \\
\%\end{array}$ & 2 & $\begin{array}{c}\% \\
10.0 \\
\%\end{array}$ & 4 & & \\
\hline
\end{tabular}

\begin{tabular}{|c|c|c|c|c|c|c|c|c|}
\hline & & Ben & $(\mathrm{N}=10]$ & & $\begin{array}{l}\text { lignant } \\
I=20 \text { ) }\end{array}$ & Tota & $\begin{array}{l}\text { Chi- } \\
\text { Square } \\
\text { Value }\end{array}$ & P-Valu \\
\hline \multirow{3}{*}{ Size } & $<2$ & 1 & $10.0 \%$ & 0 & $0.0 \%$ & 1 & \multirow{3}{*}{4.666} & \multirow{3}{*}{0.097} \\
\hline & $2-5.0$ & 7 & $70.0 \%$ & 9 & $45.0 \%$ & 16 & & \\
\hline & $>5$ & 2 & $20.0 \%$ & 11 & $55.0 \%$ & 13 & & \\
\hline \multirow{4}{*}{ Shape } & Irregular & 4 & $40.0 \%$ & 17 & $85.0 \%$ & 21 & \multirow{4}{*}{9.429} & \multirow{4}{*}{0.024} \\
\hline & Multilobulated & 0 & $0.0 \%$ & 1 & $5.0 \%$ & 1 & & \\
\hline & Oval & 2 & $20.0 \%$ & 0 & $0.0 \%$ & 2 & & \\
\hline & Round & 4 & $40.0 \%$ & 2 & $10.0 \%$ & 6 & & \\
\hline \multirow{3}{*}{ Margin } & Circumscribed & 5 & $50.0 \%$ & 0 & $0.0 \%$ & 5 & \multirow{3}{*}{16.875} & \multirow{3}{*}{0.0001} \\
\hline & Indistinct & 5 & $50.0 \%$ & 7 & $35.0 \%$ & 12 & & \\
\hline & Spiculated & 0 & $0.0 \%$ & 13 & $65.0 \%$ & 13 & & \\
\hline \multirow{3}{*}{ T1 SI } & Heterogeneous & 3 & $30.0 \%$ & 1 & $5.0 \%$ & 4 & \multirow{3}{*}{60.160} & \multirow{3}{*}{0.049} \\
\hline & Hypointense & 5 & $50.0 \%$ & 18 & $90.0 \%$ & 23 & & \\
\hline & Isointense & 2 & $20.0 \%$ & 1 & $5.0 \%$ & 3 & & \\
\hline \multirow{3}{*}{ T2 SI } & Hyperintense & 6 & $60.0 \%$ & 4 & $20.0 \%$ & 10 & \multirow{3}{*}{7.832} & \multirow{3}{*}{0.020} \\
\hline & Hypointense & 3 & $30.0 \%$ & 16 & $80.0 \%$ & 18 & & \\
\hline & Isointense & 1 & $10.0 \%$ & 0 & $0.0 \%$ & 1 & & \\
\hline
\end{tabular}

\begin{tabular}{|c|c|c|c|c|c|c|c|c|}
\hline & & & $\begin{array}{l}\text { enign } \\
=10)\end{array}$ & & $\begin{array}{l}\text { Malignant } \\
(\mathrm{N}=20)\end{array}$ & Tota & $\begin{array}{l}\text { Chi- } \\
\text { Square } \\
\text { Value }\end{array}$ & P-Value \\
\hline \multirow{2}{*}{$\begin{array}{l}\text { Non mass } \\
\text { enhancemen } \\
\text { (NME) }\end{array}$} & Absent & 8 & $80.0 \%$ & 18 & $90.0 \%$ & 26 & \multirow[b]{2}{*}{0.577} & \multirow[b]{2}{*}{0.448} \\
\hline & Present & 2 & $20.0 \%$ & 2 & $10.0 \%$ & 4 & & \\
\hline \multirow{3}{*}{$\begin{array}{c}\text { Mass } \\
\text { enhancemen } \\
\text { (ME) }\end{array}$} & Heterogeneous & 2 & $20.0 \%$ & 20 & $100.0 \%$ & 22 & \multirow{3}{*}{21.818} & \multirow{3}{*}{0.0001} \\
\hline & Homogenous & 6 & $60.0 \%$ & 0 & $0.0 \%$ & 6 & & \\
\hline & RIM & 2 & $20.0 \%$ & 0 & $0.0 \%$ & 2 & & \\
\hline \multirow{3}{*}{$\begin{array}{l}\text { Kinetic curve } \\
\text { (KC) }\end{array}$} & I & 7 & $70.0 \%$ & 1 & $5.0 \%$ & 8 & \multirow{3}{*}{18.348} & \multirow{3}{*}{0.001} \\
\hline & II & 3 & $30.0 \%$ & 4 & $20.0 \%$ & 7 & & \\
\hline & III & 0 & $0.0 \%$ & 15 & $75.0 \%$ & 15 & & \\
\hline
\end{tabular}

Comparison of BIRADS Assessment on Mammography with Histopathological Diagnosis

- Mammography correctly characterized 6 out of 10 breast lesions as benign (Birads II and III). One case of granulomatous mastitis was falsely characterized as Birads V. 2 cases of fibroadenomas and one case of intraductal papilloma were falsely characterized as Birads IV.

- Mammography correctly characterized 17 out of 20 lesions as malignant breast lesions (BIRADS IV and V). 3 cases of intraductal carcinoma were falsely characterized as benign breast lesion.

- Architectural distortion was seen in only 2 out of 10 patients (20\%) with benign lesions on mammography as compared to 18 / 20 patients with malignant lesions (90\%).
- Skin thickening was seen in only 1 out of 10 benign lesions which was seen in one case of granulomatous mastitis while skin thickening was present in 11 out of 20 malignant breast lesions (45\%).

- $\quad$ One benign lesion showed nipple retraction while it was present in 10 out of 20 malignant lesions.

\section{Diffusion Properties and ADC Values of Benign Breast Lesion}

- Out of 10 benign breast lesions 8 lesions did not show diffusion restriction on diffusion weighted imaging.

- 2 cases of granulomatous mastitis showed diffusion restriction.

- The least ADC value was $0.98 \times 10^{-3} \mathrm{~mm}^{2} / \mathrm{s}$ seen in granulomatous mastitis while maximum ADC value was $2.2 \times 10^{-3} \mathrm{~mm}^{2} / \mathrm{s}$ seen in case of fibroadenoma.

- The mean ADC value for benign breast lesion was $1.59 \mathrm{x}$ $10^{-3} \mathrm{~mm}^{2} / \mathrm{s}$.

Diffusion Properties and ADC Values of Malignant Breast Lesions

- All the malignant breast lesions show diffusion restriction on diffusion weighted imaging.

- 15 out of 20 malignant lesions had ADC values below $1 \mathrm{x}$ $10^{-3} \mathrm{~mm}^{2} / \mathrm{s}$.

- The least ADC value was $0.6 \times 10^{-3} \mathrm{~mm}^{2} / \mathrm{s}$ seen in intraductal carcinoma.

- While the maximum ADC value was $1.21 \times 10^{-3} \mathrm{~mm}^{2} / \mathrm{s}$ seen in intraductal carcinoma.

- The mean ADC value was calculated as $0.88 \times 10^{-3} \mathrm{~mm}^{2} /$ $\mathrm{s}$ for malignant breast lesions.

Comparison of Diffusion Restriction on Benign and Malignant Lesions

- 8 out of 10 benign lesions did not show restricted diffusion on DWI while 2 cases of granulomatous mastitis showing restricted diffusion on DWI, The mean ADC value calculated in benign lesion was 1.59

- All the malignant lesions show restricted diffusion on DWI with mean ADC value calculated as 0.88

Comparison of BIRADS Assessment on MRI with Histopathology.

- $\quad$ MRI correctly characterized $9 / 10$ benign lesions as Birads II / III while one case of granulomatous mastitis was characterized as BIRADS IV.

- MRI correctly characterized all malignant lesions as Birads IV / V

\begin{tabular}{|ccc|}
\hline Statistics & Value & $\mathbf{9 5} \% \mathbf{C I}$ \\
\hline Sensitivity & $95.00 \%$ & $75.13 \%$ to $99.87 \%$ \\
\hline Specificity & $90.00 \%$ & $55.50 \%$ to $99.75 \%$ \\
\hline Positive likelihood ratio & 9.5 & 1.48 to 61.16 \\
Negative likelihood ratio & 0.06 & 0.01 to 0.38 \\
\hline Disease prevalence $\left(^{*}\right)$ & $66.67 \%$ & $47.19 \%$ to $82.71 \%$ \\
Positive predictive value $\left({ }^{*}\right)$ & $95.00 \%$ & $74.69 \%$ to $99.19 \%$ \\
\hline Negative predictive value $\left(^{*}\right)$ & $90.00 \%$ & $56.85 \%$ to $98.40 \%$ \\
\hline Accuracy $\left({ }^{*}\right)$ & $93.33 \%$ & $77.93 \%$ to $99.18 \%$ \\
\hline Table 4. Diagnostic Performance of Diffusion Weighted Imaging \\
\hline
\end{tabular}




\begin{tabular}{|ccc|}
\hline Statistics & Value & 95 \% CI \\
\hline Sensitivity & $95.00 \%$ & $75.13 \%$ to $99.87 \%$ \\
Specificity & $70.00 \%$ & $34.75 \%$ to $93.33 \%$ \\
Positive likelihood ratio & 3.17 & 1.22 to 8.21 \\
Negative likelihood ratio & 0.07 & 0.01 to 0.50 \\
Disease prevalence $\left({ }^{*}\right)$ & $66.67 \%$ & $47.19 \%$ to $82.71 \%$ \\
Positive predictive value $\left({ }^{*}\right)$ & $86.36 \%$ & $70.97 \%$ to $94.26 \%$ \\
Negative predictive value $\left(^{*}\right)$ & $87.50 \%$ & $49.82 \%$ to $98.01 \%$ \\
\hline Accuracy $\left({ }^{*}\right)$ & $86.67 \%$ & $69.28 \%$ to $96.24 \%$ \\
\hline Table 5. Diagnostic Performance of Dynamic Contrast Enhanced MRI \\
\hline
\end{tabular}

\section{DISCUSSION}

The present study was undertaken to evaluate the role of MRI in characterizing benign and malignant breast lesions in diffusion weighted imaging and dynamic contrast enhancement MRI and to correlate these findings with pathological diagnosis. A total of 30 female patients presenting with palpable lumps were included in the study. In the study five patients presented with multiple lesions.

\section{Mammography}

The most common shape seen in benign lesions were either round or oval which were accounting for 6 out of 10 benign breast lesions (60\%). ${ }^{5}$ Out of 10 benign breast lesions showed well circumscribed margins. Our findings were similar to those of Evans et al. ${ }^{6}$ who reported that features seen in majority of benign lesions on mammography were round, oval, low density or slightly lobulated with welldefined margins. ${ }^{6}$ Out of 10 benign lesions shows low density on mammography. Our result was similar to that by Evans et al. ${ }^{6}$ who reported that majority of benign breast lesions showed low density on mammography.

In this study 18 out of 20 malignant lesions showed irregular shape with 11 out of 20 malignant lesions showing spiculated margins on mammography. 18 out of 20 malignant lesions showed high density on mammography. This result is in concordance with Woods et al. ${ }^{7}$ who also concluded that high density, irregular shape and spiculated margins were significantly associated with the probability of malignancy.

In our study calcification was seen in 4 out of 10 benign lesions ( $40 \%$ ) while 19 out of 20 malignant lesions shows calcification on mammography. Yunus et al. ${ }^{8}$ stated that clustered microcalcifications were significantly associated with malignancy.

In our study architectural distortion was observed as an associated finding in 13 out of 20 malignant cases (65\%). Other associated features of malignancy like skin thickening, nipple retraction and axillary lymphadenopathy were seen in $55 \%, 40 \%$ and $80 \%$ of the cases respectively. The study conducted by Sickles ${ }^{9}$ found that almost $20 \%$ of the cancers were detected primarily by indirect mammographic signs of malignancy, such as focal architectural distortion, asymmetry and developing density sign.

\section{Morphology of Breast Lesions on MRI}

6 out of 10 benign breast lesions ( $60 \%$ ) were either round or oval in shape. 5 out of 10 benign breast lesions were well circumscribed margins. Our results are in concordance with
Hockman et al.10 who observed that 19 out of 23 fibroadenomas were lobular, oval or round in shape.

In our study 5 out 10 benign lesions showed hypointense signal on T1WI, while 6 out of 10 benign lesions showed hyperintense signal on T2WI. Our result was in concordance with Westra et al. ${ }^{11}$ who reported that most masses with high signal intensity at T2WI were benign.

In our study 17 out of 20 malignant breast lesions (85\%) had irregular shape and 13 out of 20 malignant lesions (65 $\%)$ showed spiculated margins while 7 out of 20 malignant lesions (35\%) showed indistinct margins. Our findings are similar to those reported by Gutierrez et al. ${ }^{12}$ who found that larger mass size, irregular shape and irregular or spiculated margins were associated with higher odds of malignancy than smaller, smooth marginated masses.

In our study 18 out of 20 malignant lesions showed hypointense signal on T1WI while 16 out of 20 malignant lesions showed hypointense signal on T2WI. This result was in concordance with a study by Westra et al. ${ }^{11}$ who reported that most malignant lesions do not show high signal intensity on T2WI because of their high cellularity and low water content.

\section{Enhancement Pattern and DCE-MRI}

All the benign breast lesions showed enhancement on post contrast scans. The most common enhancing pattern was homogenous enhancement which was seen in 6 out of 10 benign breast lesions ( $60 \%$ ). Guiterrez et al. ${ }^{12}$ also pointed that lesion measuring more than $1 \mathrm{~cm}$ in size and showing homogenous enhancement was more likely to be benign.

All the malignant breast lesions in this study showed heterogenous enhancement. Pinker-Domenig et al. ${ }^{13}$ also observed that heterogenous enhancement was positively associated with malignancy. Gutierrez et al. ${ }^{12}$ also concluded that heterogeneous enhancement was a strong predictor of malignancy.

In our study 7 out of 10 benign lesions showed type I dynamic curve enhancement $(70 \%)$ followed by 3 benign lesions showing type II dynamic curve. On the other hand, 15 out of 20 malignant breast lesions showed type III dynamic curves (75\%) followed by 4 / 20 lesions showing type II dynamic curve. One case of IDC showed type I curve. Our findings are in concordance with Pinker-Domenig et al. ${ }^{13}$ who stated that the final diagnosis of malignancy was positively associated with type III dynamic curve. In our study P-value for DCE-MRI is 0.001

In our study the sensitivity and specificity of DCE-MRI for the detection and characterization of breast lesions was calculated as $95 \%$ and $70 \%$ respectively. Our results are comparable to those of Peters et al. ${ }^{14}$ who performed a metaanalysis to determine the diagnostic performance of contrast material enhanced magnetic resonance imaging in patients with breast lesions and calculated a pooled sensitivity of 90 $\%$ and specificity of $72 \%$.

\section{Diffusion Weighted Imaging}

In our study 8 out 10 benign lesions did not show restricted diffusion on DWI. The mean ADC value among the benign lesion was $1.59 \times 10^{-3} \mathrm{~mm}^{2} / \mathrm{s}$. All the malignant breast lesions showed restricted diffusion on DWI. The mean ADC 
value for malignant was $0.88 \times 10^{-3} \mathrm{~mm}^{2} / \mathrm{s}$. These values were well in correlation with the results of Woodhams et al. ${ }^{15}$ in whose study the mean ADC value of benign lesions was $1.67+/-0.54 \times 10^{-3} \mathrm{~mm}^{2} / \mathrm{s}$ and of malignant lesions was $1.22+/-0.31 \times 10^{-3} \mathrm{~mm}^{2} / \mathrm{s}$

In our study the sensitivity and specificity of diffusion weighted imaging to differentiate between benign and malignant lesions were $95 \%$ and $90 \%$ respectively. The corresponding PPV and NPV were $90.91 \%$ and $100 \%$ respectively. Our results were similar to that of Abdul Ghaffar et al. ${ }^{16}$ who found that DWI was $95.4 \%$ sensitive and $97.5 \%$ specific.

In our study the cut off value of ADC to differentiate between benign and malignant lesions was calculated to be $1.19 \times 10^{-3} \mathrm{~mm}^{2} / \mathrm{s}$ using ROC curve. This yielded in a sensitivity of $90 \%$ and specificity of $95 \%$. In comparison, Tan et al. ${ }^{17}$ calculated the cut off ADC values for benign and malignant lesions as $1.21 \times 10^{-3} \mathrm{~mm}^{2} / \mathrm{s}$ for $\mathrm{b}=500 \mathrm{~s} / \mathrm{mm}^{2}$ and $1.22 \times 10^{-3} \mathrm{~mm}^{2} / \mathrm{s}$ for $\mathrm{b}=1000 \mathrm{~s} / \mathrm{mm}^{2}$, respectively. In their study, the sensitivity of DCE-MRI alone was $100 \%$ with a specificity of $66.7 \%$. When DCE-MRI was combined with b $=1000 \mathrm{~s} / \mathrm{mm}^{2}$, the specificity rose to $100 \%$ while only mildly affecting sensitivity (90.6\%).

\section{Comparison of Mammography and MRI for Diagnosis of Breast Lesions}

Mammography correctly categorised 6 out of 10 lesions as benign (Birads - II / III), one case of granulomatous mastitis was characterized as BIRADS V while one case of intraductal papilloma and two cases of fibroadenoma were falsely characterized as BIRADS IV. On MRI they showed either type I or II dynamic curve, although two cases of granulomatous mastitis showed restricted diffusion on DWI.

Mammography correctly characterized (85\%) 17 out of 20 lesions as malignant (BIRADS IV / V). 3 cases were falsely characterized as benign breast lesion These lesions were correctly characterized on MRI as BIRADS $\mathrm{V}$ showing restricted diffusion. All the malignant lesions were correctly characterized as BIRADS 5 on MRI. Thus, MRI could assess the probability of malignancy in these lesions more accurately than mammography.

Liberman et al. ${ }^{18}$ assessed the positive predictive value of mammographic features and final assessment categories described in the BIRADS and correlated with the biopsy results. They observed that of the 492 lesions subjected to biopsy, BIRADS final assessment categories were category 3 in eight lesions (2\%), category 4 in 355 (72\%) and category 5 in 129 (26\%). The frequency of carcinoma was higher in category 5 than in category 4 lesions for all mammographic lesion types.

\section{Combined Analysis of DCE-MRI and DWI}

We did a combined analysis of DCE-MRI and DWI to differentiate between benign and malignant breast lesions. The individual sensitivity of DCE-MRI and DWI was $95 \%$ which remained $95 \%$ when a positive result from any of the modality was accepted as malignancy. While specificity of DWI and DCE-MRI was $90 \%$ and $70 \%$ respectively which increased to $80 \%$ when a positive result from any of the modality was accepted as malignancy.
Our results were similar to those of Tezca, Ozturk, Uslu et al. ${ }^{3}$ where sensitivity of DCE-MRI and DWI was calculated to be $100 \%$ and $92 \%$ respectively while the specificity of DCEMRI and DWI was calculated to be $59.4 \%$ and $95 \%$ respectively. Combined analysis of both DCE-MRI and DWI gave a sensitivity and specificity of $100 \%$ and $81 \%$ respectively.

\section{CONCLUSIONS}

MR morphology, DCE-MRI and DWI are useful to characterise various breast lesions. MRI features of signal intensity of hypointensity on T2WI with other associated features of irregular shape, spiculate margins, heterogeneous enhancement on DCE-MRI, type III dynamic curve and reduced $A D C$ value are strong predictors of malignancy.

Data sharing statement provided by the authors is available with the full text of this article at jemds.com.

Financial or other competing interests: None.

Disclosure forms provided by the authors are available with the full text of this article at jemds.com.

\section{REFERENCES}

[1] Button T, Dulaimy K, Fisher P, et al. Addition of MRS and perfussion MRI to conventional dynamic contrast MRI improves specificity in detection of breast malignancy. Proct Int Soc Magn Reson Med 2002.

[2] Saslow D, Boetes C, Burke W, et al. American Cancer Society guidelines for breast screening with MRI as an adjunct to mammography. CA Cancer J Clin 2007;57(2):75-89.

[3] Tezcan S, Ozturk FU, Uslu N, et al. The role of combined diffusion-weighted imaging and dynamic contrastenhanced mri for differentiating malignant from benign breast lesions presenting washout curve. Can Assoc Radiol J 2020:846537120907098.

[4] Partridge SC, McDonald ES. Diffusion weighted MRI of the breast: protocol optimization, guidelines for interpretation and practical clinical application. Magn Reson Imaging Clin North Am 2013; 21(3):601-62.

[5] Pereira FPA, Martins G, Figueiredo E, et al. Assessment of breast lesions with diffusion-weighted MRI: comparing the use of different b values. AJR Am J Roentgenol 2009; 193(4):1030-5.

[6] Evans WP. Breast masses. Appropriate evaluation. Radiol Clin North Am 1995; 33(6):1085-108.

[7] Woods RW, Sisney GS, Salkowski LR, et al. The mammographic density of a mass is a significant predictor of breast cancer. Radiology 2011; 258(2):41725.

[8] Yunus M, Ahmed N, Masroor I, et al. Mammographic criteria for determining the diagnostic value of microcalcifications in the detection of early breast cancer. J Park Med Assoc 2004; 54(1):24-9.

[9] Sickles EA. Mammographic features of 300 consecutive nonpalpable breast cancers. AJR Am J Roetengenol 1986; 146(4):661-3. 
[10] Hockman MG, Orel SG, Powell CM, et al. Fibroadenomas: MR imaging appearance with radiologic-histopathologic correlation. Radiology 1997; 204(1):123-9.

[11] Westra C, Dialani V, Mehta TS, et al. Using T2-weighted sequences to more accurately characterize breast masses seen on MRI. AJR Am J Roentgenol 2014; 202(3):W183-90.

[12]Gutierrez RL, DeMartini WB, Eby PR, et al. BI-RADS lesion characteritics predict likelihood of malignancy in breast MRI for masses but not for nonmass like enhancement. AJR Am Roentgenol 2009; 193(4):9941000.

[13] Pinker-Domenig K, Bogner W, and Gruber S, et al. Highresolution MRI of the breast at 3T: which BIRADS descriptors are most strongly associated with the diagnosis of breast cancer? Eur Radiol 2012; 22(2):32230 .
[14] Peters NHGM, Rinkes IHMB, Zuithoff NPA, et al. Metaanalysis of MR imaging in the diagnosis of breast lesions. Radiology 2008; 246(1):116-24.

[15] Woodhams R, Matsunaga K, Kan S, et al. Adc mapping of breast and malignant breast tumors. Magn Reson Med Sci 2005; 4(1):35-42.

[16] Abdulghaffar W, Tag-aldeen MM. Role of DiffusionWeighted Imaging (DWI) and Apparent Diffusion Coefficient $(A D C)$ in differentiating between benign and malignant breast lesions. The Egyptian Journal of Radiology and Nuclear Medicine 2013; 44(4):945-51.

[17] Tan H, Li R, Peng W, et al. Radiological and clinical features of adult non-puerperal mastitis. Br J Radiol 2013; 86(1024):20120657.

[18] Liberman L, Abramson AF, Squires FB, et al. The breast imaging reporting and data system: positive predictive value of mammographic features and final assessment categories. AJR Am J Roentgenol 1998; 171(1):35-40. 